\title{
Verständnis der Pathophysiologie hilft bei der individuellen Therapie der PsA
}

Das klinische Erscheinungsbild der Psoriasis Arthritis ist komplex. Daher kann ein umfassenderes Wissen um die pathophysiologischen Zusammenhänge nützlich sein, um die Frühzeichen zu lesen, Risikofaktoren für den Übergang zur systemischen Erkrankung beziehungsweise für eine Chronifizierung zu erkennen und letztlich eine individuell maßgeschneiderte Therapie initiieren zu können.

Quelle: Dr. Wiebke Kathmann, SpringerMedizin.de
Ein Verständnis der Pathophysiologie kann zu einer besseren Patientenversorgung führen, wie dem Vortrag von Prof. Dr. Georg Schett, Innere Medizin 3 (Rheumatologie und Immunologie), Universitätsklinikum Erlangen der Friedrich Alexander-Universität Erlangen-Nürnberg, im Rahmen einer „From bench to bedside"-Sitzung der EULAR (European League Against Rheumatism) zu entnehmen war. Relativ bekannt ist, dass die Interleukin(IL)-23/ IL-17-Achse maßgeblich am pathophysiologischen Geschehen bei einer Plaque-Psoriasis (PsO) und PsoriasisArthritis (PsA) beteiligt ist und sowohl über das adaptive, als auch das angeborene Immunsystem aktiviert wird.

\section{Der Übergang von der Plaque- Psoriasis zur Psoriasis-Arthritis} Bekannt ist auch, dass etwa 30-40\% der PsO-Patienten im Verlauf eine Gelenkbeteiligung entwickeln. Was genau in dieser Übergangsphase geschieht, warThema von Schetts Vortrag. Die psoriatische Erkrankung wird demnach durch ein mechanisches Trauma angestoßen, beispielsweise durch schwere manuelle Arbeit oder eine Gelenkverletzung. Voraussetzung dafür, dass der pathologische Prozess in Gang kommt, ist eine genetische Disposition des Psoriasis-Patienten.

》) Die Psoriasis wird durch ein mechanisches Trauma wie eine Gelenkverletzung angestoßen In Analogie zum Köbner-Phänomen der Haut kommt es zum so genann- ten tiefen Köbner-Phänomen an den Enthesen. Das enthesiale Stadium mit Entzündung der Enthesen gilt heute als Übergangsstadium von der Hautzur Gelenkerkrankung, also der PsO zur PsA.

\) Voraussetzung dafür, dass der pathologische Prozess in Gang kommt, ist eine genetische Disposition

Entscheidend für den Übergang ins chronische Stadium sind Autoimmunvorgänge. Klinisch lässt sich die Gelenkbeteiligung im Sinne einer subklinischen Synovitis beziehungsweise einer subklinischen Enthesiopathie laut Schett schon früh nachweisen. Sie führt zum "Homing“ der Entzündung in muskuloskeletalen Strukturen.

\section{Konsequenzen für die Therapie?}

Ob es sinnvoll ist, PsO-Patienten daher bereits im Stadium der subklinischen Synovitis mit einem IL-17 A-Inhibitor $z u$ behandeln, zumal wenn sie unspezifische Gelenkschmerzen aufweisen, hat die Arbeitsgruppe von Schett in einer kleinen Studie untersucht. Wie Dr. Eleni Kampylafka beim EULAR berichtete, wurden 20 Patienten mit PsO-Diagnose und Bedarf für eine systemische Therapie in die prospektive, offene „proof of concept“-Studie IVEPSA (Interception in Very Early PsA) eingeschlossen.

$70 \%$ von innen waren Männer, im Median 49,5 Jahre alt und seit 14 Jah-

Median waren 10,9\% der Körperoberfläche betroffen. $55 \%$ wiesen eine $\mathrm{Na}$ gel-, $75 \%$ eine Kopfhautbeteiligung und $85 \%$ Arthralgien auf (VAS-PainScore 18,5). In der körperlichen Untersuchung gaben $40 \%$ schmerzhafte Gelenke an (10 TJC - Tender Joint Count, O SJC - Swollen Joint Count).

In der Bildgebung der dominanten Hand fand sich bei $83,3 \%$ im MRT $\geq 1$ entzündliche Läsion $(66,7 \%$ Synovitis, 55,6\% Tendinitis/Enthesitis). In der hochauflösenden peripheren quantitativen Computer-Tomographie (HRpQCT) konnten bei 58,8\% Erosionen und bei $41,2 \%$ Enthesiophyten nachgewiesen werden.

》) Das enthesiale Stadium gilt heute als Übergangsstadium von der Hautzur Gelenkerkrankung

Nach sechs subkutane Gaben von je $300 \mathrm{mg}$ Secukinumab (Woche 0, 4, 8, $12,16,20$ ) hatten sich Gelenkschmerzen und Hautsymptome am Studienende nach 24 Wochen signifikant gebessert. Zudem waren in MRT und HRpQCT keine neuen Erosionen und Proliferationen nachweisbar. Laut Kampylafka legen die Daten nahe, dass das Unterbrechen des Krankheitsprozesses im Stadium der prä-PsA möglich ist und sinnvoll sein kann.

rheuma plus $2018 \cdot 17: 148$ https://doi.org/10.1007/s12688 018-0203-1

(c) Springer-Verlag GmbH Austria, ein Teil ren erkrankt. Ihr medianer PASI (Psoriasis Area Severity Index) betrug 6,8. Im 